\title{
DIPEPTIDE ADSORPTION ON Si(100)-2 × 1 ASYMMETRIC SURFACE BY FIRST PRINCIPLES
}

\author{
ETHEM AKTÜRK \\ UNAM-Institute of Material Science and Nanotechnology \\ Bilkent University, Bilkent, Ankara, Turkey \\ OĞUZ GÜLSEREN \\ Department of Physics, Bilkent University \\ Bilkent, Ankara, Turkey \\ HANDAN ARKIN \\ Department of Physics Engineering, Ankara University \\ Tandogan, Ankara, Turkey \\ TARIK ÇELIK \\ Turkish Academy of Sciences \\ Piyade Sok. No. 27, Çankaya, Ankara, Turkey \\ tcelik@tuba.gov.tr
}

Received 26 June 2009

Accepted 23 October 2009

\begin{abstract}
The adsorption of alanine dipeptide on a $\operatorname{Si}(100)-2 \times 1$ asymmetric surface is studied by using pseudopotential plane wave approach based on Density Functional Theory (DFT). Adsorption energies for different surface sites of various conformations are calculated and the groove site is found to be energetically most favorable. We observed that the molecule-surface interactions might modify surface reconstruction: asymmetric surface dimers reconstruct to asymmetric dimers in opposite directions doubling the surface periodicity, which in turn gives the surface metallic properties.
\end{abstract}

Keywords: Peptide adsorption; $\operatorname{Si}(100)-2 \times 1$ surface; Density Functional Theory.

PACS Nos.: 68.43.Bc, 68.47.Fg, 68.47.Pe.

\section{Introduction}

Recent developments in nanotechnology have made it possible to control the arrangement of biomaterials over semiconductor surfaces and opened a new stage of biotechnology combined with advanced electronics. Increasing efforts are currently being devoted to tailoring the structural and chemical properties of peptide adsorption on metal and semiconductor surfaces. Applications in a number 
of emerging technological fields, from heterogeneous catalysis to microelectronic devices and sensor developments, e.g. "DNA biochips," pose novel specific requirements in terms of surface geometry, which may be produced by bare surfaces via appropriate functionalization with organic molecules. Although a detailed knowledge of the adsorption scenario is essential to develop controlled functionalization, the atomic scale features of peptides on inorganic surfaces is still a relatively young field of research. Recent experiments of the adsorption of short peptides at semiconductor surfaces have been reported that surface structure as well as different amino acid sequences strongly affect the binding properties of these peptides at surfaces. ${ }^{1-3}$ The theoretical treatment of the adsorption of peptides at surfaces has been also a long standing problem that still gains a lot of interest. $^{4,5}$

Considerable number of experimental and theoretical investigation so as to elucidate the structural properties of such systems appeared in the literature. Early studies were performed on small molecules such as acetylene, ${ }^{6}$ ethylene ${ }^{7}$ and propylene. ${ }^{8}$ Later on, ethylene hydrogen co-adsorption was investigated as a contribution on the debate on dimer breakage upon adsorption. ${ }^{9}$ More recently, larger molecules as well as clusters have been employed in such studies. This gave rise to investigation of methanol, cyclopentene and ethanol adsorption on silicon. ${ }^{10-12}$ Those studies give a wider picture of the adsorption process, for instance, the energetics of the process as a function of the reaction coordinates. ${ }^{13,14}$ The simple amino acid glycine adsorption was investigated on several surfaces $\mathrm{Cu}, \mathrm{TiO}_{2}, \mathrm{Si}, \mathrm{NiAl}, \mathrm{Au}$, $\mathrm{Pt}$ and single-walled carbon nanotubes. ${ }^{15-26}$ Cysteine adsorption on $\mathrm{Si}(001)$ and $\mathrm{Au}(110)$ surface were investigated by Cucinotta ${ }^{27}$ and Kühnle. ${ }^{28}$ The adsorption of small peptides, for example dipeptides on $\mathrm{Si}(100)-2 \times 1$ asymmetric surface has not been thoroughly investigated by using density functional theory.

The experimental equipment has reached to such high resolutions allowing for precise identification of single molecule shapes at the surface, and the available computational capacities with sophisticated algorithms makes it possible to examine the problem, step by step, of a hybrid interface between biological molecule and semiconductor surface.

The aim of the present work is to provide a detailed description of the structural and energetic features of the adsorption of alanine dipeptide, a protein which consists of two alanine amino acids connected by a peptide bond, on the $\operatorname{Si}(100)-2 \times 1$ asymmetric surface from $a b$ initio calculations within the pseudopotential plane wave method based on the Density Functional Theory (DFT). A complete picture of the surface interaction with the peptide can be obtained by defining the possible adsorption sites. Depending on the adsorption energy, the adsorption of the dipeptide might be selective towards one or more of these sites. The present work also aims at calculating the adsorption energy for different sites in order to establish the existence of a preferential adsorption site and the possibility of geometrical distortion or reconstruction of surface as a result of the adsorption. 
The structure of this paper is as follows. Section 2 presents the computational details, Sec. 3 displays the findings and their analysis, and Sec. 4 presents concluding remarks.

\section{Computational Details}

The process of adsorption of alanine dipeptide on $\operatorname{Si}(100)-2 \times 1$ has been investigated by means of first-principle calculations within the Local Density Approach (LDA) to density functional theory, using plane wave basis with the "PWSCF" code. ${ }^{29}$ For all calculations, we have used Perdew-Zunger LDA exch-corr Vanderbilt ultrasoft pseudopotentials. ${ }^{30}$ The electronic wave functions were expanded in terms of plane waves with kinetic energy cut-off up to $340 \mathrm{eV}$. The Brillouin-zone summation was sampled by $1 \times 1 \times 1$ and $2 \times 4 \times 1 k$-point of Monkhorst grid. Atomic relaxation was taken into account via the calculations of the forces acting on the atoms in the plane wave formalism. In this calculation, the system was fully relaxed until Hellmann-Feynman forces were smaller than $0.001 \mathrm{Ry} /$ bohr. The $\mathrm{Si}(100)-2 \times 1$ asymmetric surface was modeled by a slab geometry which is periodically repeated in [100] and [010] direction, with a $(4 \times 4)$ surface unit cell. The supercell included an atomic slab with six layers of $\mathrm{Si}$ atoms, with vacuum level about $10 \AA$ in the (001) direction. The lower layer atoms in the slab were saturated with hydrogen atoms. Finally, the initial stable conformation of alanine dipeptide was obtained using the multicanonical algorithm. ${ }^{31}$

\section{Results and Discussions}

To investigate the dipeptide adsorption on the $\mathrm{Si}(100)-2 \times 1$ asymmetric surface, various adsorption sites are considered. There are four different high symmetry adsorption sites: top, bridge, groove and groove-bridge. All these sites, as well as the other minimum energy positions that resulted from the scanning of the surface by the molecule without geometry optimization at first try, are checked for different molecule orientations over the surface. Surface structure is described by six layers of slab with asymmetric dimer reconstruction. Bottom two layers of the slab are kept fixed and all the other Si atoms as well as all the atoms of the adsorbate molecule are relaxed for geometry optimization.

It is well known that the clean $\operatorname{Si}(001)$ surface exhibits $c(4 \times 2)$ and $p(2 \times$ 2) reconstructions at low temperatures and the $(2 \times 1)$-reconstruction at room temperature. $^{32}$ On the other hand, the adsorption of molecules on surface and the vibrational properties of molecules were always investigated by using several experimental methods at room temperature ${ }^{33}$ while the available theoretical results are at zero temperature. To reveal the effects of dipeptide adsorption on silicon surface at room temperature, $(2 \times 1)$-reconstruction is the starting point for our calculations.

In this work, we demonstrate that DFT may unravel the bonding of amino acids on $\operatorname{Si}(100)-2 \times 1$ asymmetric surface and provides a clear discernment between the 
reconstruction of surface dimers and their preservation upon amino acids adsorption.

The adsorption energy $E_{\text {ads }}$ is calculated as:

$$
E_{\text {ads }}=E_{\text {surf }+ \text { mol }}-E_{\text {surf }}-E_{\text {mol }}
$$

where $E_{\text {surf }}$ is the total energy of the $\mathrm{Si}_{96} \mathrm{H}_{16}$ surface (in their optimal geometry), $E_{\text {mol }}$ is the total energy of alanine dipeptide, and $E_{\text {surf }+ \text { mol }}$ is the total energy of alanine dipeptide and $\mathrm{Si}_{96} \mathrm{H}_{16}$ surface together. We calculated all the energies by substituting the system to relax by having all atoms reach into their minimum energy positions at temperature $T=0 \mathrm{~K}$. The adsorption energies are calculated for several orientations of the molecule and for different sites. The most favorable six chemical adsorption configurations are shown in Fig. 1 and the calculated adsorption energies are presented in Table 1. As it can be seen from Table 1, the groove site was found to be the most energetically favorable adsorption site for the adsorption of alanine dipeptide on $\operatorname{Si}(100)-2 \times 1$ surface. The most stable equilibrium configuration, which is obtained after geometrical optimization, is represented in Fig. 1(a). The adsorption energy of this site is $\left(E_{\text {ads }}=-2.23 \mathrm{eV}\right)$, which is characterized by the formation of a covalent bond between $\mathrm{Si}$ and $\mathrm{O}$ atoms, and the calculated length of $\mathrm{Si}-\mathrm{O}$ bond is $1.78 \AA$. These values can be compared with the tabulated one: $1.63 \AA$ ( $\mathrm{Si}-\mathrm{O}$, in $\mathrm{SiO}_{2}$ ). The $\mathrm{Si}-\mathrm{O}$ bond is slightly elongated with respect to that of bulk silica, as could be expected for a surface bond. In another study dealing with the adsorption of methanol molecule on $\mathrm{Si}(100)-2 \times 1$ surface, the calculated Si-O bond is $1.76 \AA{ }^{10}$ These results are also in good agreement with our values.

The second energetically favorable state is the groove- $180^{\circ}$ which is obtained by rotating around the $\mathrm{CH}_{3}$ axis by $180^{\circ}$ [shown in Fig. 1(b)]. In this case, the other oxygen atom is bonded to the other $\mathrm{Si}$ atom of the considered surface site. The calculated binding energy of this configuration is $-2.04 \mathrm{eV}$ and the calculated Si-O bond length is $1.80 \AA$. Comparing with the groove site, the $\mathrm{Si}-\mathrm{O}$ bond length is a little extended. As shown in Fig. 1(c), the system can also assume a configuration characterized by a bond between the surface and the oxygen atom. In this case, the occurrence of unsaturated dangling bonds giving rise to partially filled bands leads

Table 1. Calculated adsorption energies for the alanine dipeptide on $\operatorname{Si}(100)-2 \times 1$ surface.

\begin{tabular}{lc}
\hline Conformation & Adsorption energy $(\mathrm{eV})$ \\
\hline groove site & -2.23 \\
groove-180 & -2.04 \\
y-bridge & -1.66 \\
plane1 & -1.67 \\
plane2 & -1.93 \\
plane3 & -1.33 \\
\hline
\end{tabular}




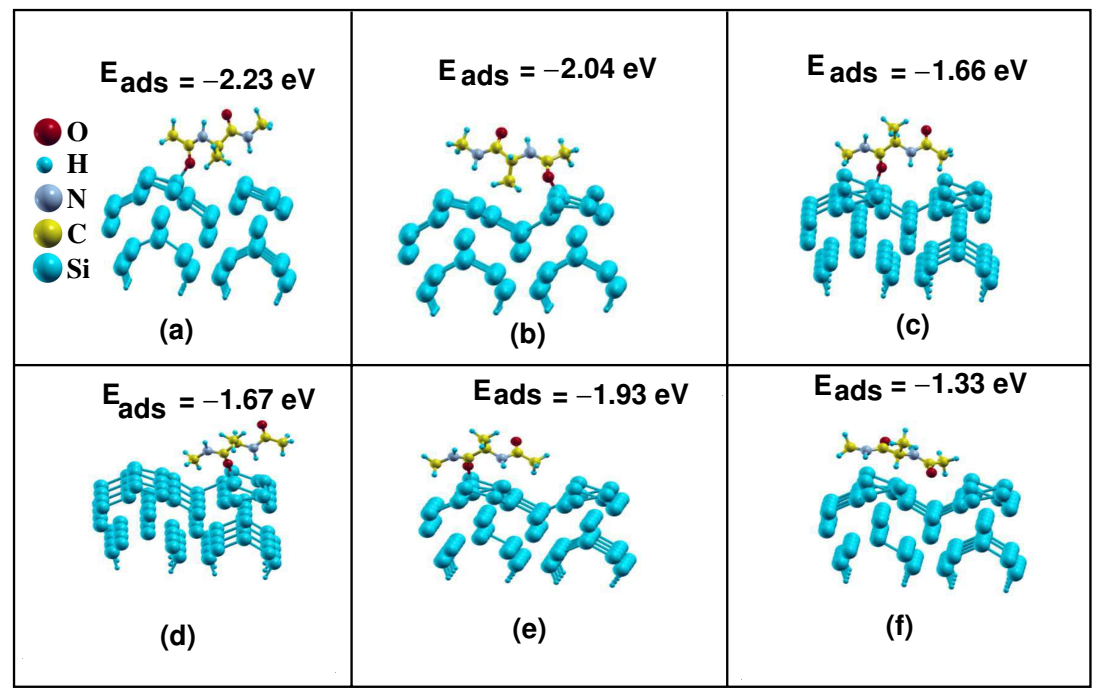

Fig. 1. (Color online) Panels (a)-(f) show the chemical bonding configurations and corresponding binding energies for chemisorption of a alanine dipeptide on a silicon substrate. (a) Calculated alanine dipeptide adsorbed on groove, (b) groove- $180^{\circ}$, (c) y-bridge, (d) plane1, (e) plane2, and (f) plane3 site of $\mathrm{Si}(100)$ surface.

Table 2. Summary of obtained Si-O bond length and to compar with experimental value.

\begin{tabular}{lcc}
\hline \multicolumn{3}{c}{ Si-O bond length } \\
\hline \multicolumn{1}{c}{ Conformation } & Calculated & Experimental $\left(\mathrm{SiO}_{2}\right)$ \\
\hline groove [see Fig. 1(a)] & 1.78 & \\
groove-180 A [see Fig. 1(b)] & 1.80 & \multirow{2}{*}{1.63} \\
y-bridge [see Fig. 1(c)] & 1.80 & \\
plane1 [see in Fig. 1(d)] & 1.82 & \\
plane2 [see in Fig. 1(e)] & 1.81 & \\
\hline
\end{tabular}

to a new reconstruction of a surface. Hence, the dangling bonds are saturated when the amino acid is adsorbed. The adsorption energy $\left(E_{\text {ads }}=-1.66 \mathrm{eV}\right)$ is smaller than for the groove and groove- $180^{\circ}$ cases and the bond length is same as for the groove- $180^{\circ}$ site (see Table 2 ).

The other two configurations which are called plane1 and plane 2 are also forming Si-O bonds. The bond lengths are 1.82 and $1.81 \AA$, respectively. They are longer than the corresponding $\mathrm{Si}-\mathrm{O}$ bond for the adsorption on a groove site (a difference of 0.4 and $0.3 \AA$ ). The adsorption energies are $E_{\text {ads }}=-1.67 \mathrm{eV}$ and $-1.93 \mathrm{eV}$ for these configurations, respectively. One can conclude from these results, with the energy value of $E_{\mathrm{ads}}=-2.23 \mathrm{eV}$, the groove site is the most probable adsorption site. 
The last equilibrium configuration is depicted in Fig. 1(f) (plane3), which results from chemisorption reactions involving the alanine dipeptide. In this case, the chemisorption energy is $-1.33 \mathrm{eV}$ and a $\mathrm{Si}-\mathrm{O}$ bond is not formed. Consequently, all the Si-O bond lengths obtained for different adsorption sites are listed in Table 2. The results for the Si-O bond lengths are in good agreement with the previous experimental and theoretical results for other systems which also form Si-O bonds. ${ }^{10}$

To support our results given above that the interaction of alanine dipeptide with Si(100)- $2 \times 1$ surface cause to bonding between Si and O atoms, we have depicted the electron density map for one of the adsorption sites (for the most probable adsorption site) in Fig. 2. The accumulation of the charge in the Si-O bonding region can be seen from the charge density difference

$$
\Delta n(r)=n_{\text {peptide } / \text { surface }}(r)-\left[n_{\text {surface }}(r)+n_{\text {peptide }}(r)\right],
$$

where $n_{\text {peptide/surface }}(r), n_{\text {surface }}(r)$ and $n_{\text {peptide }}$ are the electron charge density distributions of the peptide on surface, clean surface and peptide, respectively. In the small insert picture of Fig. 2, the isolines at the middle correspond to the region where the difference is negative, indicating there is lowering in the electron density. The isolines at the bottom part of the picture are positive showing the regions where there is an increment of electron density. The analysis leads us to realize that there is an electron density increment in the $\mathrm{Si}-\mathrm{O}$ interatomic region indicating the formation of a covalent bond.

In order to see the influence of dipeptide adsorption on surface, we have calculated the surface electronic band structure for the surface and for the dipeptide-surface system for different adsorption sites. In Fig. 3 we show the results in reciprocal space. The band structure of the asymmetric dimer structures of the surface are depicted in Fig. 3(a). The asymmetric surface is semiconducting, and our results are in good agreement with previous calculations. ${ }^{34-36}$ The separation of the occupied and unoccupied bands associated with the asymmetric dimer is found as $0.17 \mathrm{eV}$. Further, we focus on the electronic band structures of the dipeptide/Si(100)-2 $\times 1$ system given in Fig. 3(b) for groove site, (c) for groove- $180^{\circ}$ and (d) for $\mathrm{y}$-bridge site. As seen in Fig. 3, the adsorption of the dipeptide on different sites gives rise to dramatic changes on the electronic structure. Therefore, the adsorption can be conformation-selective. Firstly, when the adsorption of dipeptide on the surface occurred at groove site, because of the localization of band within the bulk valence band, the electron transferred from surface to molecule. As a result of this, the electron transfer decreases the ionicity and increases the covalency of the asymmetric dimer. Therefore, the band structure of groove site is similar to clean surface. Secondly, the calculated band structures of the dipeptide adsorption on semiconductor surface in the groove- $180^{\circ}$ site are depicted in Fig. 3(c). The surface of this structure includes many quantum confinement states or localized states resulting from occupied surface states. On the other hand, the rotation of dipeptide molecule also reduces the molecular interaction and, consequently, dispersion is developed in the surface band 


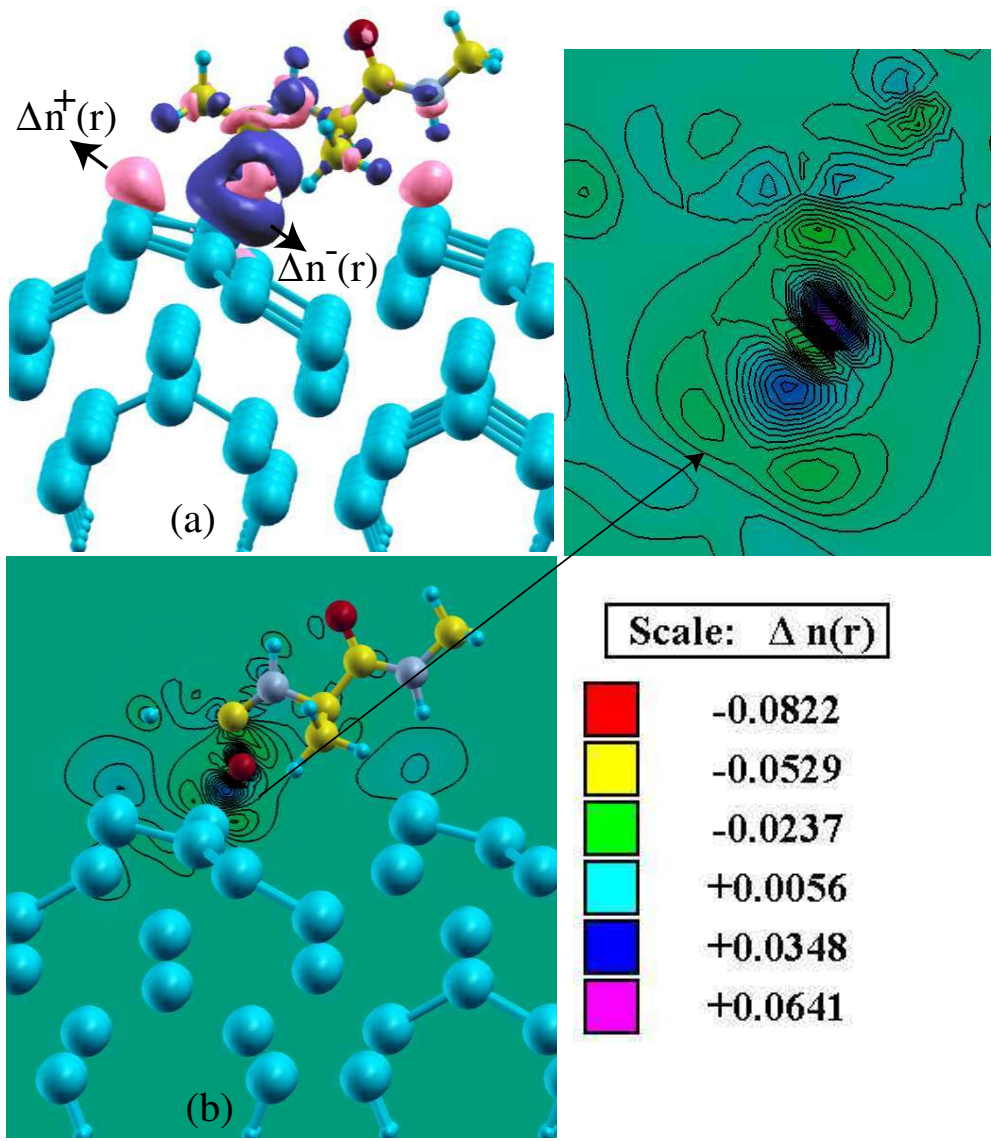

Fig. 2. (Color online) (a) Isosurfaces of the different electron charge density. $\Delta n^{+}(r)$ with pink and $\Delta n^{-}(r)$ blue isosurface showing charge accumulation and charge depletion regions respectively. Isosurface charge density is taken to be 0.003 electrons $/ \AA^{3}$. (b) The electron density maps for alanine dipeptide adsorbed on groove of Si surface atoms obtained from periodic LDA DFT calculation.

when compared with the clean surface band structure [Fig. 3(a)]. When we compare the adsorption energies, the difference between the energies associated with groove and groove- $180^{\circ}$ is relatively small, although the electronic structures are very different from each other. In groove- $180^{\circ}$ case, a distortion occurs and consequently the energy level of surface state shows a splitting due to Jahn-Teller effect. In groove case the $\pi$ dimer states interactions are not strong enough to cause a splitting of surface states and to open up a surface gap. The difference in electronic structure is also explained by using Chadi's ${ }^{37}$ predictions that the bonding and antibonding $\pi$ dimer states have separated in energy when dimer buckles occur at groove- $180^{\circ}$. Although the bare surface has indirect band gap, the surface band gap was reduced by $0.1 \mathrm{eV}$ at groove- $180^{\circ}$ and system becomes direct band gap. In addition, the formation of antisymmetric dimer at the 


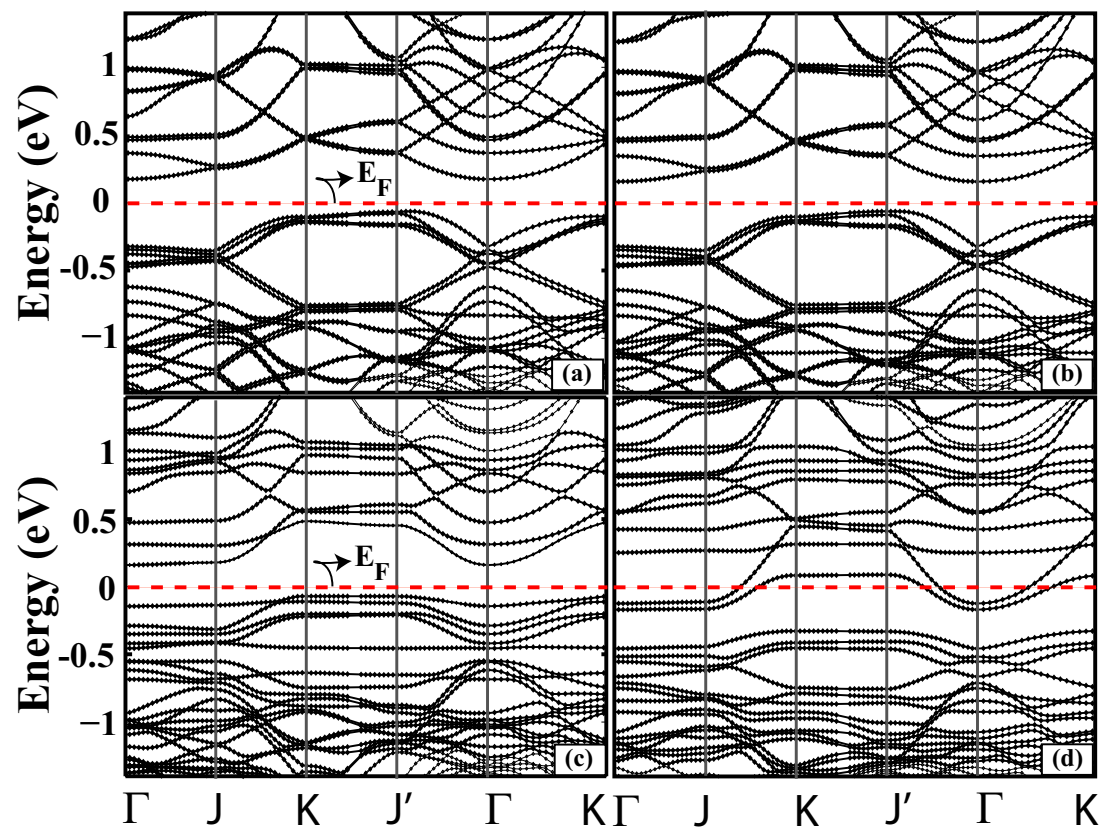

Fig. 3. (a) The energy band structure of bare Si surface folded to the $(4 \times 4)$ unit cell, (b) energy band structure of groove, (c) groove- $180^{\circ}$ and y-bridge site. The zero band energy is set to Fermi Energy of $E_{F}$. The points $\Gamma, \mathrm{J}, \mathrm{K}$, and $J^{\prime}$ are described as in Ref. 34 .

interaction region results in a charge transfer from down atom to up atom at groove$180^{\circ}$.

Finally, the band structure of the dipeptide adsorption in the y-bridge site are calculated and presented in Fig. 3(d). An interesting situation comes out when one examines the band structure. The O-terminated dimer is tilded due to the interaction between the molecules and topmost atoms of surface and thus dimer becomes asymmetric due to the charge transfer from down to up dimer atom. As a result of this, the surface bands differ from the bulk bands at the middle of the band gap, $\pi$ and $\pi^{*}$ bands overlap which in turn makes the surface gain metallic property. These electronic band structure changes are also reflected in structural modifications of the Si surface dimers. In short, the adsorption of dipeptide on semiconductor surface in $y$-bridge site changes the electronic properties of the surface upon adsorption.

\section{Conclusions}

A theoretical investigation using the DFT method has been carried out in the study of alanine dipeptide adsorption process on $\mathrm{Si}(100)-2 \times 1$ asymmetric surface. All probable adsorption sites over the surface and all probable molecule orientations are searched in detail. The geometrical optimization of the alanine dipeptide adsorbed on various sites was found to lead different adsorption energies (being measures of the stability of the adsorbate-substrate system). There are different favorable 
adsorption sites, of which the groove site is found to be the energetically most favorable. Our results have shown that the adsorption of dipeptide is undissociative and new Si-O bond is created upon adsorption. Previous experimental and theoretical studies of methanol adsorption on the same surface also shows that after the process new Si-O bonds are formed. Our calculated Si-O bond lengths for differ-

ent sites are also in good agreement with previous works. ${ }^{10}$ Finally, an interesting situation comes out when we examine the band structure. The adsorption of the dipeptide on $\mathrm{Si}(100)-2 \times 1$ asymmetric surface in $y$-bridge site changes the surface electronic properties, where the surface bands overlap with the bulk conduction bands. This in turn gives the surface metallic character. This is an important result, since it is generally expected that selective synthetic peptides may play an essential role in future nanotechnological applications.

\section{Acknowledgments}

Tarik Çelik acknowledges The Turkish Academy of Sciences. Handan Arkin acknowledges support by The Scientific and Technological Research Council of Turkey under the project number $104 \mathrm{~T} 150$ and The Turkish Academy of Sciences under the program to Reward Successful Young Scientists.

\section{References}

1. S. R. Whaley et al., Nature (London) 405, 665 (2000).

2. K. Goede, P. Busch and M. Grundmann, Nano Lett. 4, 2215 (2004).

3. K. Goede et al., AIP Conf. Proc. 893, 611 (2007).

4. M. Bachmann and W. Janke, Phys. Rev. Lett. 95, 058102 (2005).

5. M. Bachmann and W. Janke, Phys. Particles Nucl. Lett. 5, 243 (2008).

6. A. J. Dyson and P. V. Smith, Surf. Sci. 194, 55 (1997).

7. C. Huang, W. Widdra and W. H. Weinberg, Surf. Sci. 312, L953 (1994).

8. B. Craig, Surf. Sci. 298, 87 (1993).

9. R. Miotto, A. C. Ferraz and G. P. Srivastava, Phys. Rev. B 65, 075401 (2002).

10. M. Carbone and K. Larsson, J. Phys.: Condens. Matter 17, 1289 (2005).

11. P. L. Silvestrelli et al., Phys. Rev. B 68, 235306 (2003).

12. J.-H. Cho and L. Kleinman, Phys. Rev. B 64, 235420 (2001).

13. X. Lu, Q. Zhang and M. C. Lin, Phys. Chem. Chem. Phys. 3, 2156 (2001).

14. P. L. Silvestrelli, Surf. Sci. 552, 17 (2004).

15. V. Efstathiou and D. P. Woodruff, Surf. Sci. 531, 304 (2003).

16. Y.-Q. Qu et al., Surf. Sci. 569, 12 (2004).

17. L. M. Ghiringhelli, P. Schravendijk and L. DelleSite, Phys. Rev. B 74, 035437 (2006).

18. A. C. Ferraz and R. Miotto, Brazilian J. Phys. 36, 2A (2006).

19. G. Jones et al., Surf. Sci. 600, 1924 (2006).

20. R. B. Rankin and D. S. Sholl, J. Chem. Phys. 124, 074703 (2006).

21. M. Nydberg et al., J. Chem. Phys. 112, 5420 (2000).

22. S. M. Barlow et al., Surf. Sci. 401, 322 (1998).

23. E. Soria et al., Surf. Sci. 451, 188 (2000).

24. A. Lopez et al., Chem. Phys. 277, 1 (2002).

25. X. Y. Xiao et al., Langmuir 18, 6274 (2002).

26. T. Roman et al., Thin Solid Films 509, 218 (2006). 
27. C. S. Cucinotta et al., Phys. Rev. B 72, 245310 (2005).

28. A. Kuhnle et al., Phys. Rev. Lett. 93, 086101 (2004).

29. S. Baroni et al., http://www.pwscf.org (2001).

30. D. Vanderbilt, Phys. Rev. B 41, R7892 (1990).

31. B. A. Berg and T. Çelik, Phys. Rev. Lett. 69, 2292 (1992).

32. J. Dabrowski and H.-J. Müssig, Silicon Surfaces and Formation of Interfaces (World Scientific, Singapore, 2000).

33. M. D. Ellison and R. J. Hamers, J. Phys. Chem. B 103, 6243 (1999).

34. A. Ramstad, G. Brocks and P. J. Kelly, Phys. Rev. B 51, 14 (1995).

35. J. Ihm, M. L. Cohen and D. J. Chadi, Phys. Rev. B 21, 4592 (1980).

36. M. Rohlfing, P. Krüger and J. Pollmann, Phys. Rev. B 52, 13753 (1995).

37. D. J. Chadi, Phys. Rev. Lett. 43, 47 (1979). 